\title{
Developmental perspectives on primate gesture: 100 years in the making
}

\author{
Erica A. Cartmill ${ }^{1}$ Catherine Hobaiter ${ }^{2,3}$
}

Published online: 5 July 2019

○) Springer-Verlag GmbH Germany, part of Springer Nature 2019

\begin{abstract}
This paper is an introduction to the special issue entitled Evolving the study of gesture: evaluating and unifying theories of gesture acquisition in great apes. The gestures of great apes have been recorded in scientific literature for over 100 years, but the ways in which apes acquire their gestures remains a highly debated topic. Through this historical framework, we summarize and contextualize contemporary research on the development of ape gesture. We describe the papers presented in this special issue, grouping them into three themes: assessing theories, methodological innovation, and new empirical approaches. Each of the papers is a significant contribution to the literature on ape gesture, but the collection of work together represents a unique collaboration across labs, theories, and studied species. By considering the papers side-by-side, we hope that readers will see the authors as engaging in a true dialogue, one which will help the field of primate gesture research make significant advances in the years to come.
\end{abstract}

\section{History of ape gesture research}

The gestures of great apes have been a subject of scientific curiosity for nearly 150 years, but the development of ape gestures remains woefully understudied. Early descriptions from the late nineteenth and early twentieth centuries are often highly detailed, and many conclusions early scholars drew about the 'nature' of ape gestures are strikingly prescient, with studies a century later coming to similar conclusions.

One of the first scientific descriptions of ape gesture occurs in Darwin's (1872) book, The expression of emotions in man and animals. Although the book focuses most closely on facial expression and vocalizations, Darwin records several gestural displays in great detail. In one passage, Darwin describes a juvenile female chimpanzee's display of distress and notes the similarity with behavior seen in human children:

Erica A. Cartmill

cartmill@anthro.ucla.edu

1 Department of Anthropology, University of California, Los Angeles, Box 951553, Los Angeles, CA 90095, USA

2 School of Psychology and Neuroscience, University of St Andrews, St Andrews, Scotland KY16 9JP, UK

3 Budongo Conservation Field Station, P.O. Box 362, Masindi, Uganda
"A young female chimpanzee, in a violent passion, presented a curious resemblance to a child in the same state...She screamed loudly with widely open mouth, the lips being retracted so that the teeth were fully exposed. She threw her arms wildly about, sometimes clasping them over her head. She rolled on the ground, sometimes on her back, sometimes on her belly, and bit everything within reach.” (Darwin 1872: p 140)

Darwin describes the gestures of apes as more 'expressive' than their facial expressions, but goes on to question whether the facial expressions of monkeys might not be more expressive still, owing to their more highly marked coloration and their control over ear movements. We now believe that the flexibility with which apes use gesture distinguishes gesture from other signals, such as facial expression (Call 2007; Tanner and Byrne 1993), and Darwin's interest in 'expressiveness' has no equivalent in contemporary studies. Current literature on ape gesture focuses on intentionality, gesture modification, and meaning. Features that might contribute to what Darwin referred to as expressiveness-like the relative size or salience of signals—are rarely remarked upon in current work, unless perhaps as a feature that apes manipulate in selecting or modifying their signals to be more easily perceived by others (e.g. Cartmill 2009).

In the early twentieth century, scientists observing chimpanzees again compared their gestures to other modes of communication and concluded that their gestures appeared 
to be more flexibly used and less reflective of the apes' inner emotional states than their vocalisations were (Rothmann and Teuber 1915; Yerkes and Learned 1925). In comparing chimpanzee communication to that of humans, Rothmann and Tuber (1915) argued that:

"...as a means of intercommunication, vocalisation is less important and bodily attitude and gesture more important in the chimpanzee than in man." (Rothmann and Teuber 1915, pp. 13-14)

Wolfgang Kohler, writing about a series of studies he conducted on tool-use in chimpanzees in 1914-1915, describes several gestures in detail. His description of a "wrist present" used for appeasement resonates with our contemporary understanding of the gesture:

"When a chimpanzee approaches another of the same species with whom he is on a "difficult" footing-for instance, if they have recently been fighting - and is dubious about the possible reception of his advances, he will probably extend his hand with the palm turned inwards...one may perhaps guess that the flexion of the palm and the extension of the back of the hand are meant to reassure, by contrast to the grasping or hacking motion characteristic of attack." (Kohler 1925, p 305)

Kohler was one of the first researchers to note that although chimpanzee gestures are remarkably expressive and display considerable variation, they are not used to label objects in the way that human language does. These early observations of ape gesture were anecdotal and involved small numbers of animals, but the authors spent many hours with their subjects, and many of the comparisons they made between ape and human communication have, to a large extent, been supported by recent systematic studies of ape gesture.

\section{Studies of ape development and gesture}

Although Darwin noted the similarities between gestural and facial displays in apes and human children, studies of ape development were not conducted until the early twentieth century. In the 1920s, Robert Yerkes and Blanche Learned detailed the cognitive and communicative ontogeny of two young chimpanzees housed first in Washington D.C., and then in Cuba (Yerkes and Learned 1925). They described the chimpanzees' physical, cognitive, and vocal development. Yerkes and Learned focused on vocal communication, rather than gestural or facial signals, transcribing their subjects' vocalizations using musical notation and giving great detail about the contexts in which they occurred. The authors noted the chimpanzees' facial expressions and gestural movements while describing their attempts (and frustrations) in communicating vocally with their human caretakers.

In the 1930s, Nadezhda Ladygina-Kohts published the first direct comparison of development in a chimpanzee and a human child (Ladygina-Kohts and de Waal 2002). She raised the chimpanzee in her home as a human child (foreshadowing later work on enculturated apes), and documented the first 3 years of life in great detail, later comparing them to her own son's development. Building on Darwin's work, Ladygina-Kohts employed a photomontage technique to document and compare subtle details in the facial expressions of her two subjects. She noted similarities and differences in the expressions produced by the human and chimpanzee during similar interactions, such as tickling. Her descriptions and illustrations of chimpanzee gesture were highly detailed and the gestures she described appear in largely similar forms in contemporary work. When describing the chimpanzee's reaction to being shown a dead magpie, Ladygina-Kohts writes:

"...the chimpanzee turned his side to it, bared his teeth, covered his face with one hand, and made a hesitant waving gesture with the other." (Ladygina-Kohts and de Waal 2002: p 104)

This early work on ape gesture and communicative development involved apes in captivity, but by the middle of the twentieth century, the gestures and calls of wild apes began to be systematically described (Schaller 1963; van LawickGoodall 1968). Jane Goodall conducted a longitudinal study of chimpanzees at Gombe in the late 1960s (van LawickGoodall 1967), but communicative development in wild apes was not systematically studied until the work of Frans Plooij a decade later (Plooij 1978, 1979, 1984).

Although gesture was often described in broader studies of ape behavior or communication, it wasn't until the 1980s that ape gesture itself became the focus of scientific research, starting with the work of Michael Tomasello and colleagues at the Yerkes Primate Research Center (Tomasello et al. 1985, 1989, 1994, 1997). These studies by Tomasello and colleagues had an explicit focus on development, but answers to the questions they raised about gesture ontogeny remain elusive. The authors employed both cross-sectional and longitudinal designs to study gesture in chimpanzees, arguing that gesture was more flexible than other forms of communication and that it demonstrated relatively sophisticated forms of social cognition. After moving to the Max Planck Institute for Evolutionary Anthropology in Leipzig in the late 1990s, Michael Tomasello and Josep Call launched a program of comparative gesture studies with the goal of studying gesture in multiple primate species using the same methods. This program included work on gorillas, bonobos, orangutans, and siamangs (Pika et al. 2003, 2005; Liebal et al. 2004, 2006), and established many of the 
coding standards shared across the field today. Most of the studies in the MPI's comparative gesture project focused on the gestures of juvenile apes, but they did not explicitly look at development. Instead, they documented the gestures produced by apes in multiple captive groups and compared repertoires between sites. These studies reported that apes' gestures did not differ systematically between sites, and the authors concluded that ape gestures were probably not acquired via cultural transmission.

In the early 2000s, a group of researchers at the University of St Andrews began another series of comparative gesture studies, focusing largely on the repertoires of gesture types and the meanings of ape gestures (e.g., Genty et al. 2009; Cartmill and Byrne 2010; Hobaiter and Byrne 2011a). These studies built upon the work of the MPI group, but also drew on Richard Byrne and Joanne Tanner's detailed studies of social cognition and forms of reference in the gestures of captive gorillas (Tanner and Byrne 1993, 1996). Early work in the group described the large repertoires of gestures available within ape species (Genty et al. 2009; Hobaiter and Byrne 2011a; Graham et al. 2017), and how these were deployed and combined (Genty et al. 2009; Cartmill and Byrne 2010; Hobaiter and Byrne 2011b). They employed the cognitive approach to ask what signalers 'mean' when using a gesture. Because ape gesture, like language, was shown to be directed towards specific recipients with a particular goal, it was possible to explore more than simply the 'information' encoded within a signal, and ask instead what the signaler intended to communicate: gestural meaning (Cartmill and Byrne 2010; Hobaiter and Byrne 2014; Graham et al. 2018). The group also moved the systematic study of gesture back into the field, providing the first descriptions of wild ape gesture that explored the full repertoires of gestures available to, first, chimpanzees (Hobaiter and Byrne 2011a, b), and then bonobos (Graham et al. 2018), and most recently orangutans (Knox et al., in press).

Today, the picture that emerges from the field is largely one of consensus: great apes employ large repertoires of their gestures flexibly and intentionally to achieve day-to-day goals while navigating their social world. However, the question of gesture ontogeny, first raised a century ago by Yerkes and Learned, remains unresolved. A diverse range of mechanisms have been suggested, from genetic channeling to ritualization to social learning, and with the expansion of the field has come a diversification of methods, making direct comparisons of data in the published literature challenging.

\section{Goal of the special issue}

The idea for this special issue began as a symposium at the 2014 Congress of the International Primatological Society in Vietnam. The goal of the symposium was to bring ape gesture researchers together to discuss questions of acquisition and ontogeny-topics that have remained lacunae in the otherwise rapidly growing literature on primate gesture. Much of the interest in primate gesture has been driven by an interest in gesture's role in the origins of human language and the argument that ape gesture shares more features in common with human language than ape vocalization does. The argument for a gesture 'first' hypothesis is weakened by recent studies showing primate vocalizations to be more flexibly deployed than previously thought (e.g., Schel et al. 2013; Crockford et al. 2017). However, language-precursor systems in our hominin ancestors (like any modern species' communication, including human language) likely employed both vocal and gestural signals. Thus, the interest in gesture's role in the evolution of human language - and, therefore, interest in primate gestures-has remained strong.

Ape gestures display many of the hallmarks of human language. They are directed towards specific individuals. They take into account whether the interlocutor can perceive them. They are repeated, modified, or combined with other signals when they do not receive a desired response. They are used to achieve specific goals. They are used flexibly and in seemingly intentional ways to achieve those goals. Multiple gestures can be used to achieve the same goal and individual gestures can be used to achieve multiple goals. However, ape gestures do not seem to be acquired as conventions in the same way that children learn new words. Apes in different groups (in both captive and wild populations) who have not interacted with one another produce gestures that are remarkably similar in both form and function. There is some individual and local variation, but not to the degree one would expect if apes acquired their gestures through cultural transmission (Tomasello et al. 1997; Hobaiter and Byrne 2011a). Apes can easily learn to perform new manual movements, so the learning problem here is not one of manual dexterity or control.

It is not clear how apes acquire their gestures, or indeed whether the acquisition process can be characterized by a single learning mechanism. The extant theories about acquisition are typically discussed as if they were irreconcilable, but this may not necessarily be true, particularly as some studies focus on different levels of explanation (e.g., proximate or ultimate; Fröhlich and Hobaiter 2018). However, although many papers discuss acquisition, very few have been able to directly study the emergence or spread of new gestures. The field of primate gesture research has grown to include substantial methodological diversity, and we may be nearing a point at which it will be possible to create an integrated theory of gesture acquisition.

This special issue takes first steps in that direction by bringing together 31 scholars across 10 papers to consider the current data on gesture acquisition and how we might be able to better study acquisition in the future. This group 
of authors encompasses the vast majority of scholars currently working on ape gesture. The collection of papers also covers a wide range of methods, from categorical coding of spontaneous interactions, to experimental games, to conversation analysis, to models of neural activation. Although each paper stands on its own as a significant contribution to the literature on primate communication, we hope that by assembling the papers into a single issue, the different perspectives, datasets, and methodological approaches will be brought into conversation with one another in ways that will provoke new insights and inspire future work. While all the papers contain multiple themes, we have organized the volume into three sections: assessing theories, methodological innovation, and new empirical approaches.

\section{Overview}

The papers in Assessing Theories (Tomasello and call 2018; Byrne et al. 2017; Liebal et al. 2018) review the existing literature on ape gesture and assess support for the two primary theories of gesture acquisition: ontogenetic ritualization and genetic channeling. Ontogenetic ritualization (OR) proposes that actions are ritualized into gestures over many successive interactions (Tomasello et al. 1994). According to $\mathrm{OR}$, individuals begin to recognize and respond to another ape's actions during the initial movements, and the acting ape learns that only part of the action is necessary to achieve their goal. Thus, over repeated interactions, the acting ape becomes the signaling ape. The main alternative theory of acquisition proposes that most gesture types are part of a species' innate repertoire. Genetic channeling (often called phylogenetic ritualization) posits that apes are born with the tendency to develop a particular set of basic gesture movements, and isolated populations of apes show little variability in the available gesture forms or repertoires (Hobaiter and Byrne 2011a). This theory doesn't imply, however, that there is no learning involved; apes may learn aspects of when and how to use their gestures and may learn to modify them in many ways. Of course, it is possible (perhaps likely) that not all gestures are acquired via the same mechanism. This possibility is discussed in several of the papers as well as the conclusion to the special issue.

In their paper, Thirty years of great ape gestures, Michael Tomasello and Josep Call review the body of work produced by their research group: starting with the studies of chimpanzee gesture at Emory in the 1980s, through the large scale cross-species comparisons conducted at the MPI in Leipzig. They discuss points of agreement and disagreement between different studies, most notably with Richard Byrne and colleagues on acquisition, and David Leavens and colleagues on referentiality. Tomasello and Call mention gesture intentionality and sensitivity to the attentional state of the recipient as areas of great agreement across studies. Many researchers have adopted their criteria for intentionality and have found evidence of intentional gesturing across all the apes. Tomasello and Call argue that ape gestures come in two distinct types: 'intention movements' and 'attention-getters.' They review the evidence from their work and propose that they are likely acquired in different ways, with intention movements resulting from ontogenetic ritualization. They argue that ape gesture is a unique form of primate communication, and thus should not be considered very similar to either species-typical signals (as, they argue, Byrne and colleagues support), or human gestures (as, they argue, Leavens and colleagues support).

In Great ape gestures: intentional communication with a rich set of innate signals, Byrne, Cartmill, Genty, Graham, Hobaiter, and Tanner review the work on ape gesture conducted by Richard Byrne and colleagues at the University of St Andrews over the last three decades. They highlight the similarities in gesture forms and meanings, with repertoires of the available gesture types largely overlapping between populations and species, and greater overlap between more closely related species. They also discuss the differences between gesture repertoire size (large relative to other animals), and number of meanings those gestures convey (relatively small, given the number of different gestures). Byrne and colleagues argue that the forms of gestures are phylogenetically old and that the basic forms and functions are largely inherited. The authors stress that this does not mean that learning is not involved, but rather than developing an adult gestural repertoire involves a process of pruning and shaping patterns of use over time until arriving at a smaller and more specific adult set of gestures and gesture meanings.

Liebal, Schneider, and Errsen-Lembeck review data from a wide range of studies to evaluate the primary theories of gesture acquisition in their paper, How primates acquire their gestures: evaluating current theories and evidence. The goal of their paper is to provide an overview of the current state of the field and to consider how well the different proposed acquisition mechanisms account for the observed data on gesture. They lay out the predictions made by the major theories about acquisition (genetic channeling, imitation, and ontogenetic ritualization) and then review the supporting evidence for each theory. The authors argue that studies of facial expression development may help forward the discussion of gesture acquisition because their developmental trajectories differ in several important ways. Ape facial expressions emerge earlier in infancy than gestures and show little developmental change. The authors argue that ape facial expressions are likely to be more genetically determined than gestures and show less evidence of social shaping. The authors also discuss theoretical differences between research groups and argue that many of these different interpretations can be explained by different research 
designs and data collection methods. They end by stressing the need for longitudinal studies, rather than cross-sectional age comparisons, to better answer questions about gesture development and highlight the need for developmental studies that gather comparable data on different modalities.

The papers in Methodological Innovation (Leavens et al. 2017; Genty 2019; Gasser and Arbib 2019) present critiques of common methods or propose new methods for studying the development of ape gestures. In their paper, The mismeasure of ape social cognition, Leavens, Bard, and Hopkins problematize common practices of comparative cognition research, which compare the raw performance of apes and humans on cognitive tasks despite vast differences in individual life histories, interaction frameworks, and methodological design. They argue that these kinds of comparisons ignore critical differences in the psychological causes underpinning differences in observed behavior. They propose a more situated approach to studying social cognition and communication, in which individuals' learning histories and local ecological differences are taken into account.

In her paper, Vocal-gestural combinations in infant bonobos: new insights into signal functional specificity, Genty highlights combinations of vocalizations and gestures by proposing to study the development of signal meaning by asking whether calls and gestures are combined more frequently to perform particular functions. Using an example call type (the pout-moan), Genty outlines and walks through a five-step method to ask whether pout-moans are combined with gestures in ways that are functionally-specific (i.e., have particular meanings). She also asks whether the functional-specificity of these combinations changes with age. Although she focuses on qualitative analyses, Genty's data suggests that bonobos' call-gesture combinations become more functionally-specific as they age.

Gasser and Arbib take a neural computational approach to the learning question in their paper, A dyadic brain model of ape gestural learning, production and representation. They present a computational model simulating how a gesture might be ritualized through the interactions and corresponding brain activation of two interacting individuals over time. Gasser and Arbib build 'dyadic brain models' of interacting apes using neurophysiological, neuroanatomical, and behavioral data and integrating specific motivations and rewards at different stages in the model. They argue that such modeling can help test theories of gesture emergence by modeling existing datasets and making testable predictions for future empirical work.

The papers in New Empirical Approaches (Bard et al. 2017; Pika and Fröhlich 2018; Lamaury et al. 2017; Kersken et al. 2018), present new data on gesture in juvenile primates (both human and non-human). They introduce new theoretical framings and approaches for studying gesture by looking at different modalities (e.g., tactile) or conducting strictly-aligned cross-species comparative studies (with human children or monkeys).

Bard, Maguire-Herring, Tomonaga, and Matsuzawa focus on the development of a tactile gesture in their paper, The gesture 'Touch': Does meaning-making develop in chimpanzees' use of a very flexible gesture? Tactile gestures are often mentioned in studies of ape gesture, but little work has focused on understanding tactile communication. Bard et al., analyze the use of touch by three juvenile chimpanzees over a five-year period and compare it to the use of touch by adults. They find that infants and adults use the touch gesture in ways that differ in form, location, and context, but also argue that there is no systematic change in the way touch is used from infancy to adulthood. Given the high variability in form and context, the authors argue that the meaning of a touch gesture cannot be fully understood from either the form or the context alone. They argue that it is thus critical for researchers to consider contextualized use of gesture in their work.

In their paper, Gestural acquisition in great apes: The Social Negotiation Hypothesis, Pika and Frohlich analyze gestures of young wild chimpanzees. They show that gesture frequency and repertoire size are positively correlated with number of non-maternal interaction partners. According to the authors, the role of social interaction has been underestimated in the two dominant theories of gesture acquisition: genetic channeling (phylogenetic ritualization) and ontogenetic ritualization. They advocate for a form of the theory Plooij introduced in 1978-the Social Negotiation Hypothesis-which posits that ape infants transition from first interpreting and responding to the intent of the signaler during a signaling event, to later understanding that communicative behaviors may themselves be used to manipulate others. Pika and Frölich argue that this transition occurs over development and allows infants to move from responding to and maintaining interactions, to initiating them using signals that have been learned in prior interactions. They propose that, over repeated interactions, apes develop a "mutual understanding" that particular behaviors can be used communicatively to "transfer distinct information and to achieve desired goals." They urge other researchers to more carefully consider the role of social learning in ape gesture and they call for more longitudinal studies and comparative datasets to help advance our understanding of gesture acquisition.

Lamaury, Cochet, and Bourjade extend the scope of the special issue beyond the great apes and beyond spontaneous intentional communication, to study the development of joint attention behaviors in monkeys being trained to produce food requesting gestures. In their paper, Acquisition of joint attention by olive baboons gesturing toward humans, Lamaury et al. trained olive baboons (Papio anubis) to request food from human experimenters using a reaching gesture. They varied the attentional cues available to 
the baboons by having the experimenter either facing the subjects or in profile. Over time, the baboons began to look towards the experimenter's face and displayed gaze alternation between the experimenter's face and the food reward when gesturing. However, this only occurred when the baboon had full visual access to the experimenter's face (in the facing condition). The authors conclude that access to the social cues present in a partner's face is essential to baboons' developing joint attention. This work demonstrates that the consideration of visual social cues is likely widespread across primate species. Monkey gesturing is significantly less studied than ape gesturing and more work needs to be done in this area. In addition to moving outside the great ape family, this work particularly stands apart from other papers in the special issue in using a training paradigm rather than analyzing spontaneous intentional communication between conspecifics. This method can be particularly useful in testing the importance of different cues during the learning or deployment of a behavior, but the learning process in a human-led training paradigm differs significantly from the learning that occurs naturalistically between conspecifics. Baboons needed training both to acquire the requesting gesture and to learn to attend to their partners' visual state. This finding could suggest an important difference between monkeys and apes; however, the salience of cues in cross-species communication provides a methodological challenge. Thus, we may need to be cautious about generalizing to natural behavior.

Kersken, Gomez, Liszkowski, Soldati, and Hobaiter turn the analytic lens onto human primates in their paper: A gestural repertoire of 1-2 year old human children: in search of the ape gestures. They argue that, historically, human gesture and ape gesture have been studied using very different methods, and thus any comparison between them is difficult, at best. They aim to overcome this methodological divide by applying an ape coding system to the gestural communication of young human children. They present data from 13 human children aged 1-2 years-old, recorded at home or in a nursery environment. Using the same methodology Hobaiter uses when studying chimpanzees, they identified 52 distinct gestures used by human toddlers. They then compared these gestures to those in the chimpanzee repertoire and found that 46 of them were present in both species. The authors argue that there is a shared gestural repertoire available to all great apes (including humans, at least while language skills remain in development), but that is it largely overlooked or unobserved when non-comparable methods are used to study human and non-human apes.

We close the volume by taking stock of the field and looking towards the future. In the final article (Gesturing towards the future: Cognition, big data, the future of comparative gesture research), we attempt to reconcile differences between dominant theories of acquisition, highlighting areas where terminological clarity or methodological innovation could resolve apparent differences in interpretation. We end by proposing new directions for research on ape gesture, and we hope that a collected volume on this topic in 20 years-time will be able to answer many of the questions that remain.

\section{References}

Bard KA, Maguire-Herring V, Tomonaga M, Matsuzawa T (2017) The gesture 'Touch': Does meaning-making develop in chimpanzees' use of a very flexible gesture?. Anim Cogn 1-16. https://doi. org/10.1007/s10071-017-1136-0

Byrne RW, Cartmill E, GentyK E, Graham E, Hobaiter C, Tanner J (2017) Great ape gestures: intentional communication with a rich set of innate signals. Anim Cogn 1-1. https://doi.org/10.1007/ s10071-017-1127-1

Call J, Tomasello M (2007) The gestural repertoire of chimpanzees (Pan troglodytes). In: Call J, Tomasello M (eds) The gestural communication of apes and monkeys. Lawrence Erlbaum Associates, London, pp 17-39

Cartmill EA (2009) Gestural communication in orangutans (Pongo pygmaeus and Pongo abelii): a cognitive approach. (Doctoral dissertation, University of St Andrews)

Cartmill EA, Byrne RW (2010) Semantics of primate gestures: intentional meanings of orangutan gestures. Anim Cogn 13(6):793-804

Crockford C, Wittig RM, Zuberbühler K (2017) Vocalizing in chimpanzees is influence by social-cognitive processes. Sci Adv 3:e1701742

Darwin C (1872) The expression of emotion in man and animals. Murray, London

Fröhlich M, Hobaiter C (2018) The development of gestural communication in great apes. Behav Ecol Sociobiol 72(12):194

Gasser B Arbib M (2019) A dyadic brain model of ape gestural learning, production and representation. Anim Cogn. 1-16. https://doi. org/10.1007/s10071-018-1228-5

Genty E, Breuer T, Hobaiter C, Byrne RW (2009) Gestural communication of the gorilla (Gorilla gorilla): repertoire, intentionality and possible origins. Anim Cogn 12:527-546

Genty E (2019) Vocal-gestural combinations in infant bonobos: new insights into signal functional specificity. Anim Cogn 1-14. https ://doi.org/10.1007/s10071-019-01267-0

Graham KE, Furuichi T, Byrne RW (2017) The gestural repertoire of the wild bonobo (Pan paniscus): a mutually understood communication system. Anim Cogn 20:171-177

Graham KE, Hobaiter C, Ounsley J, Furuichi T, Byrne RW (2018) Bonobo and chimpanzee gestures overlap extensively in meaning. PLoS Biol 16(2):e2004825

Hobaiter C, Byrne RW (2011a) The gestural repertoire of the wild chimpanzee. Anim Cogn 14:745-767

Hobaiter C, Byrne RW (2011b) Serial gesturing by wild chimpanzees: its nature and function for communication. Anim Cogn 14(6):827-838

Hobaiter C, Byrne RW (2014) The meanings of chimpanzee gestures. Curr Biol 24:1596-1600

Kersken V, Gómez J-C, Liszkowski U, Soldati A Hobaiter C (2018) A gestural repertoire of 1- to 2-year-old human children: in search of the ape gestures. Anim Cogn 1-19. https://doi.org/10.1007/ s10071-018-1213-z

Kohler W (1925) The mentality of apes. Routledge and Kegan Paul, London 
Ladygina-Kohts NN, de Waal FBM (2002) Infant chimpanzee and human child: a classic 1935 comparative study of ape emotions and intelligence. Oxford University Press, New York

Lamaury A, Cochet H, Bourjade M (2017) Acquisition of joint attention by olive baboons gesturing toward humans. Anim Cogn. 1-9. https://doi.org/10.1007/s10071-017-1111-9

Leavens DA, BardWilliam KA, Hopkins D (2017) The mismeasure of ape social cognition. Anim Cogn 1-18. https://doi.org/10.1007/ s10071-017-1119-1

Liebal K, Pika S, Tomasello M (2004) Social communication in siamangs (Symphalangus syndactylus): use of gestures and facial expressions. Primates 45(1):41-57

Liebal K, Pika S, Tomasello M (2006) Gestural communication of orangutans (Pongo pygmaeus). Gesture 6:1-38

Liebal K Schneider C, Errson-Lembeck M (2018) How primates acquire their gestures: evaluating current theories and evidence. Anim Cogn 1-14. https://doi.org/10.1007/s10071-018-1187-x

Pika S, Liebal K, Tomasello M (2003) Gestural communication in young gorillas (Gorilla gorilla): gestural repertoire, learning, and use. Am J Primatol 60:95-111

Pika S, Liebal K, Tomasello M (2005) Gestural communication in subadult bonobos (Pan paniscus): repertoire and use. Am J Primatol 65:39-61

Pika S, Fröhlich M (2018) Gestural acquisition in great apes: the Social Negotiation Hypothesis. Anim Cogn 1-15. https://doi. org/10.1007/s10071-017-1159-6

Plooij FX (1978) Some basic traits of language in wild chimpanzees? In: Lock A (ed) Action, gesture and symbol. Academic Press, London, pp 111-131

Plooij FX (1979) How wild chimpanzee babies trigger the onset of mother-infant play. In: Bullowa M (ed) Before speech. University Press, Cambridge, pp 223-243

Plooij FX (1984) The behavioral development of free-living chimpanzee babies and infants. Praeger publishers Inc, Westport

Rothmann M, Teuber E (1915) Aus der Anthropoidenstation auf Teneriffa: Ziele und Aufgaben der Station sowie erste Beobachtungen an den auf ihr gehaltenen Schimpansen. Abhandlungen der koniglich preussischen Akademie der Wissenschaften (physikalisch-mathematische Klasse) 2:1-20
Schaller GB (1963) The mountain gorilla: Ecology and behavior. University of Chicago Press, Chicago

Schel AM, Townsend SW, Machanda Z, Zuberbühler K, Slocombe KE (2013) Chimpanzee alarm call production meets key criteria for intentionality. PLoS One 8(10): e76674

Tanner JE, Byrne RW (1993) Concealing facial evidence of mood: perspective-taking in a captive gorilla? Primates 34:451-457

Tanner JE, Byrne RW (1996) Representation of action through iconic gesture in a captive lowland gorilla. Curr Anthropol 37:162-173

Tomasello M, George BL, Kruger AC, Jeffrey M, Farrar EA (1985) The development of gestural communication in young chimpanzees. $\mathbf{J}$ Hum Evol 14:175-186

Tomasello M, Gust D, Frost GT (1989) A longitudinal investigation of gestural communication in young chimpanzees. Primates 30:35-50

Tomasello M, Call J, Nagell K, Olguin R, Carpenter M (1994) The learning and use of gestural signals by young chimpanzees: a trans-generational study. Primates 35:137-154

Tomasello M, Call J (2018) Thirty years of great ape gestures. Anim Cogn 1-9. https://doi.org/10.1007/s10071-018-1167-1

Tomasello M, Call J, Warren J, Frost GT, Carpenter M, Nagell K (1997) The ontogeny of chimpanzee gestural signals: a comparison across groups and generations. Evol Commun 1:223-259

van Lawick-Goodall J (1967) Mother-offspring relationships in freeranging chimpanzees. In: Morris D (ed) Primate ethology. Weidenfeld and Nicolson, London, pp 287-346

van Lawick-Goodall J (1968) The behavior of free-ranging chimpanzees in the Gombe stream reserve. Anim Behav Monogr $1: 161-311$

Yerkes RM, Learned BW (1925) Chimpanzee intelligence and its vocal expressions. Williams \& Wilkins, Baltimore

Publisher's Note Springer Nature remains neutral with regard to jurisdictional claims in published maps and institutional affiliations. 